TRANSACTIONS OF THE

AMERICAN MATHEMATICAL SOCIETY

Volume 360, Number 6, June 2008, Pages 2811-2823

S 0002-9947(08)04470-X

Article electronically published on January 25, 2008

\title{
SANDWICH PAIRS IN CRITICAL POINT THEORY
}

\author{
MARTIN SCHECHTER
}

\begin{abstract}
Since the development of the calculus of variations there has been interest in finding critical points of functionals. This was intensified by the fact that for many equations arising in practice the solutions are critical points of functionals. If a functional $G$ is semibounded, one can find a Palais-Smale (PS) sequence

$$
G\left(u_{k}\right) \rightarrow a, \quad G^{\prime}\left(u_{k}\right) \rightarrow 0 .
$$

These sequences produce critical points if they have convergent subsequences (i.e., if $G$ satisfies the PS condition). However, there is no clear method of finding critical points of functionals which are not semibounded. The concept of linking was developed to produce Palais-Smale (PS) sequences for $C^{1}$ functionals $G$ that separate linking sets. In the present paper we discuss the situation in which one cannot find linking sets that separate the functional. We introduce a new class of subsets that accomplishes the same results under weaker conditions. We then provide criteria for determining such subsets. Examples and applications are given.
\end{abstract}

\section{INTRODUCTION}

Many problems arising in science and engineering call for the solving of the Euler equations of functionals, i.e., equations of the form

$$
G^{\prime}(u)=0,
$$

where $G(u)$ is a $C^{1}$ functional (usually representing the energy) arising from the given data. As an illustration, the equation

$$
-\Delta u(x)=f(x, u(x))
$$

is the Euler equation of the functional

$$
G(u)=\frac{1}{2}\|\nabla u\|^{2}-\int F(x, u(x)) d x
$$

on an appropriate space, where

$$
F(x, t)=\int_{0}^{t} f(x, s) d s,
$$

and the norm is that of $L^{2}$. The solving of the Euler equations is tantamount to finding critical points of the corresponding functional. The classical approach was to look for maxima or minima. If one is looking for a minimum, it is not sufficient

Received by the editors August 14, 2005.

2000 Mathematics Subject Classification. Primary 35J65, 58E05, 49J35.

Key words and phrases. Critical point theory, variational methods, saddle point theory, semilinear differential equations. 
to know that the functional is bounded from below, as is easily checked. However, one can show that there is a sequence, called a Palais-Smale PS sequence satisfying

$$
G\left(u_{k}\right) \rightarrow a, \quad G^{\prime}\left(u_{k}\right) \rightarrow 0
$$

for $a=\inf G$. Such a sequence may not produce a critical point, but if it has a convergent subsequence, then it does. If every PS sequence for $G$ has a convergent subsequence, then we say that $G$ satisfies the PS condition. However, when extrema do not exist, there is no clear way of obtaining critical points. In particular, this happens when the functional is not bounded from either above or below. What can be used to replace semiboundedness? We shall describe an approach which is very useful in such cases. As a substitute for semiboundedness, one looks for suitable sets that separate the given functional. In other words, one looks for suitable subsets $A, B$ of a Banach space $E$, which for a given $C^{1}$ functional $G$ on $E$ satisfy

$$
a_{0}:=\sup _{A} G \leq b_{0}:=\inf _{B} G .
$$

Ideally, we would want (4) to imply that $G$ has a critical point, i.e., a point $u \in E$ such that

$$
G(u)=a \geq b_{0}, \quad G^{\prime}(u)=0 .
$$

Clearly, this is too much to ask, since even semiboundedness is not sufficient to imply the existence of a critical point. However, there are pairs of subsets such that (4) produces a Palais-Smale PS sequence

$$
G\left(u_{k}\right) \rightarrow a, \quad G^{\prime}\left(u_{k}\right) \rightarrow 0,
$$

where $a \geq b_{0}$. If $A, B$ are such that (4) always implies (6), we say that $A$ links $B$. Consequently, if $A$ links $B$ and $G$ is a $C^{1}$ functional on $E$ which satisfies (4) and the PS condition, then $G$ has a critical point satisfying (5). Linking sets exist and are described in the literature.

In the present paper, we discuss the situation in which one cannot find linking sets that separate the functional, i.e., satisfy (4). Are there weaker conditions that will imply (6)? Our answer is yes, and we find pairs of subsets such that a condition weaker than (4) produces a PS sequence. We have

Definition 1. We shall say that a pair of subsets $A, B$ of a Banach space $E$ forms a sandwich, if for any $G \in C^{1}(E, \mathbb{R})$ the inequality

$$
-\infty<b_{0}:=\inf _{B} G \leq a_{0}:=\sup _{A} G<\infty
$$

implies that there is a sequence satisfying

$$
G\left(u_{k}\right) \rightarrow c, \quad b_{0} \leq c \leq a_{0}, \quad G^{\prime}\left(u_{k}\right) \rightarrow 0 .
$$

Unlike linking, the order of a sandwich pair is immaterial, i.e., if the pair $A, B$ forms a sandwich, so does $B, A$. Moreover, we allow sets forming a sandwich pair to intersect. One sandwich pair has been studied in the past. We have (cf. [Si1], $[\mathrm{Sc} 1],[\mathrm{Sc} 2])$ :

Theorem 2. Let $N$ be a closed subspace of a Hilbert space $E$ and let $M=N^{\perp}$. Assume that at least one of the subspaces $M, N$ is finite dimensional. Let $G$ be a $C^{1}$-functional on $E$ such that

$$
m_{0}:=\inf _{w \in M} G(w) \neq-\infty
$$


and

$$
m_{1}:=\sup _{v \in N} G(v) \neq \infty .
$$

Then there are a constant $c \in \mathbb{R}$ and a sequence $\left\{u_{k}\right\} \subset E$ such that

$$
G\left(u_{k}\right) \rightarrow c, m_{0} \leq c \leq m_{1}, G^{\prime}\left(u_{k}\right) \rightarrow 0 .
$$

It follows from this that $M, N$ form a sandwich pair if one of them is finite dimensional. (Note that $m_{0} \leq m_{1}$.)

Theorem 2 has been generalized as follows (cf. [Sc4]):

Theorem 3. Let $N$ be a closed subspace of a Hilbert space $E$ and let $M=N^{\perp}$. Assume that at least one of the subspaces $M, N$ is finite dimensional. Let $G$ be a $C^{1}$-functional on $E$ such that

$$
m_{0}:=\sup _{v \in N} \inf _{w \in M} G(v+w) \neq-\infty
$$

and

$$
m_{1}:=\inf _{w \in M} \sup _{v \in N} G(v+w) \neq \infty .
$$

Then there are a constant $c \in \mathbb{R}$ and a sequence $\left\{u_{k}\right\} \subset E$ such that

$$
G\left(u_{k}\right) \rightarrow c, m_{0} \leq c \leq m_{1}, G^{\prime}\left(u_{k}\right) \rightarrow 0 .
$$

This constitutes the sum total of results of this type. To date only complementing subspaces have been considered with one of them being finite dimensional. The purpose of the present paper is to show that other sets can qualify as well.

\section{Criteria}

In this section we present sufficient conditions for sets to qualify as sandwich pairs. We have

Proposition 4. If $A, B$ is a sandwich pair and $J$ is a diffeomorphism on the entire space having a derivative $J^{\prime}$ satisfying

$$
\left\|J^{\prime}(u)^{-1}\right\| \leq C, \quad u \in E,
$$

then $J A, J B$ is a sandwich pair.

Proof. Suppose $G \in C^{1}$ satisfies

$$
-\infty<b_{0}:=\inf _{J B} G \leq a_{0}:=\sup _{J A} G<\infty .
$$

Let

$$
G_{1}(u)=G(J u), \quad u \in E .
$$

Then

$$
\begin{aligned}
-\infty<b_{0} & :=\inf _{J B} G=\inf _{J u \in J B} G(J u)=\inf _{B} G_{1} \\
\leq a_{0} & :=\sup _{J A} G=\sup _{J u \in J A} G(J u)=\sup _{A} G_{1}<\infty .
\end{aligned}
$$

Since $A, B$ form a sandwich pair, there is a sequence $\left\{h_{k}\right\} \subset E$ such that

$$
G_{1}\left(h_{k}\right) \rightarrow c, \quad b_{0} \leq c \leq a_{0}, \quad G_{1}^{\prime}\left(h_{k}\right) \rightarrow 0 .
$$


If we set $u_{k}=J h_{k}$, this becomes

$$
G\left(u_{k}\right) \rightarrow c, \quad b_{0} \leq c \leq a_{0}, \quad G^{\prime}\left(u_{k}\right) J^{\prime}\left(h_{k}\right) \rightarrow 0 .
$$

In view of (12), this implies $G^{\prime}\left(u_{k}\right) \rightarrow 0$. Thus, $J A, J B$ is a sandwich pair.

Proposition 5. Let $N$ be a closed subspace of a Hilbert space $E$ with complement $M^{\prime}=M \oplus\left\{v_{0}\right\}$, where $v_{0}$ is an element in $E$ having unit norm, and let $\delta$ be any positive number. Let $\varphi(t) \in C^{1}(\mathbb{R})$ be such that

$$
0 \leq \varphi(t) \leq 1, \varphi(0)=1
$$

and

$$
\varphi(t)=0, \quad|t| \geq 1
$$

Let

$$
F\left(v+w+s v_{0}\right)=v+\left[s+\delta-\delta \varphi\left(\|w\|^{2} / \delta^{2}\right)\right] v_{0}, \quad v \in N, w \in M, s \in \mathbb{R} .
$$

Assume that one of the subspaces $M, N$ is finite dimensional. Then $A=N^{\prime}=$ $N \oplus\left\{v_{0}\right\}, B=F^{-1}\left(\delta v_{0}\right)$ is a sandwich pair.

Proof. Define

$$
J\left(v+w+s v_{0}\right)=v+w+\left[s+\delta-\delta \varphi\left(\|w\|^{2} / \delta^{2}\right)\right] v_{0}, \quad v \in N, w \in M, s \in \mathbb{R} .
$$

Then $J$ is a diffeomorphism on $E$ with its inverse having a derivative satisfying (12). Moreover, $J A=N^{\prime}$ and $J B=M+\delta v_{0}$. Hence, $J A, J B$ form a sandwich pair as long as one of them is finite dimensional (Theorem 3). We now apply Proposition 4.

Theorem 6. Let $N$ be a finite dimensional subspace of a Banach space E. Let $F$ be a Lipschitz continuous map of $E$ onto $N$ such that $F=I$ on $N$ and

$$
\|F(g)-F(h)\| \leq K\|g-h\|, \quad g, h \in E .
$$

Let $p$ be any point of $N$. Then $A=N, B=F^{-1}(p)$ forms a sandwich pair.

Proof. Let $G$ be a $C^{1}$-functional on $E$ satisfying (7), where $A, B$ are the subsets of $E$ specified in the theorem. If the theorem is not true, then there is a $\delta>0$ such that

$$
\left\|G^{\prime}(u)\right\| \geq 3 \delta
$$

whenever

$$
b_{0}-3 \delta \leq G(u) \leq a_{0}+3 \delta .
$$

Since $G \in C^{1}(E, \mathbb{R})$, there is a locally Lipschitz continuous mapping $Y(u)$ of $\hat{E}=\left\{u \in E: G^{\prime}(u) \neq 0\right\}$ into $E$ such that

$$
\|Y(u)\| \leq 1, \quad u \in \hat{E}
$$

and

$$
\left(G^{\prime}(u), Y(u)\right) \geq 2 \delta
$$

whenever $u$ satisfies (20) (for the construction of such a map, cf., e.g., [Sc4]). Let

$$
\begin{aligned}
Q_{0} & =\left\{u \in E: b_{0}-2 \delta \leq G(u) \leq a_{0}+2 \delta\right\}, \\
Q_{1} & =\left\{u \in E: b_{0}-\delta \leq G(u) \leq a_{0}+\delta\right\}, \\
Q_{2} & =E \backslash Q_{0}, \\
\eta(u) & =\rho\left(u, Q_{2}\right) /\left[\rho\left(u, Q_{1}\right)+\rho\left(u, Q_{2}\right)\right] .
\end{aligned}
$$


It is easily checked that $\eta(u)$ is locally Lipschitz continuous on $E$ and satisfies

$$
\eta(u)=1, u \in Q_{1} ; \eta(u)=0, u \in \bar{Q}_{2} ; 0<\eta(u)<1 \text {, otherwise. }
$$

Consider the differential equation

$$
\sigma^{\prime}(t)=W(\sigma(t)), \quad t \in \mathbb{R}, \sigma(0)=u \in N,
$$

where

$$
W(u)=-\eta(u) Y(u)
$$

The mapping $W$ is locally Lipschitz continuous on the whole of $E$ and is bounded in norm by 1 . Hence by a well-known existence theorem for ordinary differential equations in a Banach space, (20) has a unique solution for all $t \in \mathbb{R}$. Let us denote the solution of (20) by $\sigma(t) u$. The mapping $\sigma(t)$ is in $C(E \times \mathbb{R}, E)$ and is called the flow generated by $W(u)$. Note that

$$
\begin{aligned}
d G(\sigma(t) u) / d t & =\left(G^{\prime}(\sigma(t) u), \sigma^{\prime}(t) u\right) \\
& =-\eta(\sigma(t) u)\left(G^{\prime}(\sigma(t) u), Y(\sigma(t) u)\right) \\
& \leq-2 \delta \eta(\sigma(t) u) .
\end{aligned}
$$

Let

$$
E_{\alpha}=\{u \in E: G(u) \leq \alpha\}
$$

I claim that there is a $T>0$ such that

$$
\sigma(T) E_{a_{0}+\delta} \subset E_{b_{0}-\delta} .
$$

In fact, we take $T>\left(a_{0}-b_{0}+\delta\right) /(2 \delta)$. Let $u$ be any element in $E_{a_{0}+\delta}$. If there is a $t_{1} \in[0, T]$ such that $\sigma\left(t_{1}\right) u \notin Q_{1}$, then

$$
G(\sigma(T) u) \leq G\left(\sigma\left(t_{1}\right) u\right)<b_{0}-\delta
$$

by (22). Hence $\sigma(T) u \in E_{b_{0}-\delta}$. On the other hand, if $\sigma(t) u \in Q_{1}$ for all $t \in[0, T]$, then $\eta(\sigma(t) u)=1$ for all $t$, and (22) yields

$$
G(\sigma(T) u) \leq G(u)-2 \delta T \leq a_{0}-2 \delta T<b_{0}-\delta .
$$

Hence (23) holds.

Let $\Omega$ be a bounded open subset of $\mathrm{N}$ containing the point $p$ such that

$$
\rho(\partial \Omega, p)>K T+\delta,
$$

where $\rho$ is the distance in $E$. If $v \in \partial \Omega$, then

$$
\|v-p\| \leq\|v-F \sigma(t) v\|+\|F \sigma(t) v-p\| .
$$

Hence,

$$
\|F \sigma(t) v-p\|>K T+\delta-t K>0, \quad v \in \partial \Omega, 0 \leq t \leq T
$$

since

$$
\|F \sigma(t) v-v\| \leq K \int_{0}^{t}\left\|\sigma^{\prime}(s) v\right\| d s \leq K t
$$

Let

$$
H(t)=F \sigma(t) .
$$

Then $H(t)$ is a continuous map of $\bar{\Omega}$ into $N$ for $0 \leq t \leq T$. Moreover, $H(t) v \neq p$ for $v \in \partial \Omega$ by (25). Hence, the Brouwer degree $d(H(t), \Omega, p)$ is defined. Consequently,

$$
d(H(T), \Omega, p)=d(H(0), \Omega, p)=d(I, \Omega, p)=1 .
$$


This means that there is a $v \in \bar{\Omega}$ such that

$$
F \sigma(T) v=p
$$

But then

$$
\sigma(T) v \in F^{-1}(p)=B \text {. }
$$

This is not consistent with (23). Hence, $A, B$ form a sandwich pair.

\section{Applications}

In the present section we assume that $\Omega$ is a bounded domain in $\mathbb{R}^{n}$ with boundary $\partial \Omega$ sufficiently regular so that the Sobolev inequalities hold and the embedding of $H^{m, 2}(\Omega)$ in $L^{2}(\Omega)$ is compact (cf. [Ad]). Let $A$ be a self-adjoint operator on $L^{2}(\Omega)$. We assume that $A \geq \lambda_{0}>0$ and that

$$
C_{0}^{\infty}(\Omega) \subset D:=D\left(A^{1 / 2}\right) \subset H^{m, 2}(\Omega)
$$

for some $m>0$, where $C_{0}^{\infty}(\Omega)$ denotes the set of test functions in $\Omega$ (i.e., infinitely differentiable functions with compact supports in $\Omega$ ) and $H^{m, 2}(\Omega)$ denotes the Sobolev space. If $m$ is an integer, the norm in $H^{m, 2}(\Omega)$ is given by

$$
\|u\|_{m, 2}:=\left(\sum_{|\mu| \leq m}\left\|D^{\mu} u\right\|^{2}\right)^{1 / 2} .
$$

Here $D^{\mu}$ represents the generic derivative of order $|\mu|$ and the norm on the right hand side of $(26)$ is that of $L^{2}(\Omega)$. We shall not assume that $m$ is an integer.

Let $q$ be any number satisfying

$$
\begin{aligned}
& 2 \leq q \leq 2 n /(n-2 m), \quad 2 m<n, \\
& 2 \leq q<\infty, \quad n \leq 2 m,
\end{aligned}
$$

and let $f(x, t)$ be a Carathéodory function on $\Omega \times \mathbb{R}$. This means that $f(x, t)$ is continuous in $t$ for a.e. $x \in \Omega$ and measurable in $x$ for every $t \in \mathbb{R}$. We make the following assumptions.

(A). The function $f(x, t)$ satisfies

$$
|f(x, t)| \leq V(x)^{q}|t|^{q-1}+V(x) W(x)
$$

and

$$
f(x, t) / V(x)^{q}=o\left(|t|^{q-1}\right) \text { as }|t| \rightarrow \infty,
$$

where $V(x)>0$ is a function in $L^{q}(\Omega)$ such that

$$
\|V u\|_{q} \leq C\|u\|_{D}, \quad u \in D
$$

and $W$ is a function in $L^{\infty}(\Omega)$. Here

$$
\begin{gathered}
\|u\|_{q}:=\left(\int_{\Omega}|u(x)|^{q} d x\right)^{1 / q}, \\
\|u\|_{D}:=\left\|A^{1 / 2} u\right\|
\end{gathered}
$$

and $q^{\prime}=q /(q-1)$. (If $\Omega$ and $V(x)$ are bounded, then (27) will hold automatically by the Sobolev inequality. However, there are functions $V(x)$ which are unbounded 
and such that (27) holds even on unbounded regions $\Omega$.) With the norm (28), $D$ becomes a Hilbert space. Define

$$
F(x, t):=\int_{0}^{t} f(x, s) d s
$$

and

$$
G(u):=\|u\|_{D}^{2}-2 \int_{\Omega} F(x, u) d x .
$$

It is readily shown that $G$ is a continuously differentiable functional on the whole of $D$ (cf., e.g., [Sc4]). Since the embedding of $D$ in $L^{2}(\Omega)$ is a compact, the spectrum of $A$ consists of isolated eigenvalues of finite multiplicity

$$
0<\lambda_{0}<\lambda_{1}<\cdots<\lambda_{\ell}<\cdots .
$$

(We take $\lambda_{0}$ to be an eigenvalue.)

Let $\lambda_{\ell}, \ell>0$, be one of these eigenvalues. We assume that the eigenfunctions of $\lambda_{\ell}$ are in $L^{\infty}(\Omega)$ and that the following hold:

$$
\begin{aligned}
2 F(x, t) \leq & \lambda_{\ell} t^{2}+W_{1}(x), \quad x \in \Omega, t \in \mathbb{R}, \text { for some } W_{1}(x) \in L^{1}(\mathbb{R}), \\
& \lambda_{\ell} t^{2} \leq 2 F(x, t), \quad|t| \leq \delta \text { for some } \delta>0, \\
\nu t^{2} \leq & 2 F(x, t), \quad x \in \Omega, t \in \mathbb{R}, \text { for some } \nu>\lambda_{\ell-1}, \\
& H(x, t):=2 F(x, t)-t f(x, t) \leq C(|t|+1)
\end{aligned}
$$

and

$$
\sigma(x):=\limsup _{|t| \rightarrow \infty} H(x, t) /|t|<0 \quad \text { a.e. }
$$

We have

Theorem 7. Under the above hypotheses,

$$
A u=f(x, u), u \in D
$$

has at least one nontrivial solution.

Proof. Let $N$ denote the subspace of $L^{2}(\Omega)$ spanned by the eigenfunctions of $A$ corresponding to the eigenvalues $\lambda_{0}, \ldots, \lambda_{\ell}$, and let $M=N^{\perp} \cap D$. Thus $D=M \oplus N$. This time we take

$$
G(u)=2 \int F(x, u) d x-\|u\|_{D}^{2},
$$

the negative of (29). We are therefore looking for solutions of $G^{\prime}(u)=0$. Let $N^{\prime}$ be the set of those functions in $N$ are orthogonal to $E\left(\lambda_{\ell}\right) . N^{\prime}$ is spanned by those eigenfunctions corresponding to $\lambda_{0}, \ldots, \lambda_{\ell-1}$. Let $v_{0}$ be an eigenfunction of $\lambda_{\ell}$ with norm one. Let $M_{1}=M \oplus E\left(\lambda_{\ell}\right) \ominus\left\{v_{0}\right\}$. We can write

$$
E=M_{1} \oplus\left\{v_{0}\right\} \oplus N^{\prime} .
$$

Consider the mapping

$$
F\left(v+w+s v_{0}\right)=w+\left[s+\delta-\delta \varphi\left(\|v\|^{2} / \delta^{2}\right)\right] v_{0}, \quad v \in N^{\prime}, w \in M_{1}, s \in \mathbb{R},
$$

where $\varphi$ satisfies the hypotheses of Proposition 5. We take

$$
A=M_{1} \oplus\left\{v_{0}\right\}, \quad B=F^{-1}\left(\delta v_{0}\right) .
$$

By Proposition 5, A, B form a sandwich pair. 
For $v \in N$, we write $v=v^{\prime}+y$, where $v^{\prime} \in N^{\prime}$ and $y \in E\left(\lambda_{\ell}\right)$. Since $E\left(\lambda_{\ell}\right)$ is finite dimensional and contained in $L^{\infty}(\Omega)$, there is a $\rho>0$ such that

$$
\|y\|_{D} \leq \rho \text { implies }\|y\|_{\infty} \leq \delta / 2
$$

where $\delta$ is given by (31). Thus, if

$$
\|v\|_{D} \leq \rho \text { and }|v(x)| \geq \delta
$$

then

$$
\delta \leq|v(x)| \leq\left|v^{\prime}(x)\right|+|y(x)| \leq\left|v^{\prime}(x)\right|+\delta / 2 .
$$

Hence

$$
|v(x)| \leq 2\left|v^{\prime}(x)\right|
$$

holds for all $x \in \bar{\Omega}$ satisfying (37). Thus by (31),

$$
\begin{aligned}
G(v) & \geq \lambda_{\ell} \int_{|v|<\delta} v^{2} d x-2 \int_{|v|>\delta}\left\{|V v|^{q}+|V v| W\right\} d x-\|v\|_{D}^{2} \\
& \geq \lambda_{\ell}\|v\|^{2}-\|v\|_{D}^{2}-C \int_{2\left|v^{\prime}\right|>\delta}\left\{\left|V v^{\prime}\right|^{q}+\left|V v^{\prime}\right| W+\delta^{2-q}\left|v^{\prime}\right|^{q}\right\} d x \\
& \geq \lambda_{\ell}\left\|v^{\prime}\right\|^{2}-\left\|v^{\prime}\right\|_{D}^{2}-C\left\|v^{\prime}\right\|_{D}^{q} \\
& \geq\left(\frac{\lambda_{\ell}}{\lambda_{\ell-1}}-1-C\left\|v^{\prime}\right\|_{D}^{q-2}\right)\left\|v^{\prime}\right\|_{D}^{2} .
\end{aligned}
$$

From this we see that there are positive constants $\epsilon, \rho$ such that

$$
G(v) \geq \epsilon\left\|v^{\prime}\right\|_{D}^{2}, \quad\|v\|_{D} \leq \rho, v \in N .
$$

Moreover, this shows that

$$
G(v) \geq \epsilon_{1}, \quad\|v\|_{D}=\rho, v \in N
$$

for some positive $\epsilon_{1}$ unless there is a solution of

$$
A y=\lambda_{\ell} y=f(x, y), y \in E\left(\lambda_{\ell}\right) \backslash\{0\}
$$

(cf. [Sc4]). Since such a solution would solve (35), we may assume that (38) holds.

Since

$$
\|v\|_{D}^{2} \leq \lambda_{\ell}\|v\|^{2}, \quad v \in N
$$

and

we have by $(30)$

$$
\lambda_{\ell+1}\|w\|^{2} \leq\|w\|_{D}^{2}, \quad w \in M
$$

$$
G(w) \leq \lambda_{\ell}\|w\|^{2}+B_{1}-\|w\|_{D}^{2} \leq B_{1}, \quad w \in A,
$$

where $B_{1}=\int_{\Omega} W_{1}(x) d x$. Moreover, (32) implies

$$
G\left(v^{\prime}\right) \geq\left(\nu-\lambda_{\ell-1}\right)\left\|v^{\prime}\right\|^{2}, \quad v^{\prime} \in N^{\prime} .
$$

Hence, there is an $\varepsilon>0$ such that

$$
G(v) \geq \varepsilon, \quad v \in B .
$$

In view of these inequalities we can now apply Proposition 5 to conclude that there is a sequence $\left\{u_{k}\right\} \subset D$ such that

$$
G\left(u_{k}\right) \rightarrow c, \varepsilon \leq c \leq B_{1}, G^{\prime}\left(u_{k}\right) \rightarrow 0 .
$$


Let $\rho_{k}=\left\|u_{k}\right\|_{D}$. If $\rho_{k} \rightarrow \infty$, then

$$
G\left(u_{k}\right)=2 \int_{\Omega} F\left(x, u_{k}\right) d x-\rho_{k}^{2} \rightarrow c
$$

and

$$
\left(G^{\prime}\left(u_{k}\right), u_{k}\right) / 2=\int_{\Omega} f\left(x, u_{k}\right) u_{k} d x-\rho_{k}^{2}=o\left(\rho_{k}\right)
$$

Hence,

$$
\int_{\Omega} H\left(x, u_{k}\right) d x=o\left(\rho_{k}\right) .
$$

Let $\tilde{u_{k}}=u_{k} / \rho_{k}$. Then $\left\|\tilde{u}_{k}\right\|_{D}=1$. Thus, there is a renamed subsequence such that $\tilde{u}_{k} \rightarrow \tilde{u}$ weakly in $D$, strongly in $L^{2}(\Omega)$ and a.e. in $\Omega$. By (33) and (34),

$$
\begin{gathered}
\limsup \int_{\Omega} H\left(x, u_{k}\right) d x / \rho_{k} \leq \int_{\Omega} \limsup \left[H\left(x, u_{k}\right) /\left|u_{k}\right|\right]\left|\tilde{u}_{k}\right| d x \\
=\int_{\Omega} \sigma(x)|\tilde{u}| d x .
\end{gathered}
$$

Since $\sigma(x)<0$ a.e. in $\Omega$, the last two statements imply that $\tilde{u} \equiv 0$. However, we see from (40) that

$$
2 \int_{\Omega} F\left(x, u_{k}\right) d x / \rho_{k}^{2} \rightarrow 1
$$

while (30) implies

$$
\limsup 2 \int_{\Omega} F\left(x, u_{k}\right) d x / \rho_{k}^{2} \leq \lambda_{\ell} \int_{\Omega} \tilde{u}^{2} d x,
$$

showing that $\tilde{u} \not \equiv 0$. This contradiction tells us that the $\rho_{k}$ must be bounded. We can now apply Theorem 3.4.1 of [Sc4] to conclude that there is a $u \in D$ satisfying

$$
G(u)=c, G^{\prime}(u)=0 .
$$

Since $c \geq \varepsilon>0$, we see that $u \neq 0$, and the proof is complete.

The proof of Theorem 7 implies

Corollary 8. If $\lambda_{\ell}$ is a simple eigenvalue, then hypothesis (30) can be weakened to

$$
2 F(x, t) \leq \lambda_{\ell+1} t^{2}+W_{1}(x), \quad x \in \Omega, t \in \mathbb{R}, \text { for some } W_{1}(x) \in L^{1}(\mathbb{R})
$$

in Theorem 7.

Remark 9. The proof of Theorem 7 is much simpler if $\ell=0$. In this case $N^{\prime}=\{0\}$ and (36) immediately implies (38). The rest of the proof is unchanged.

We now show that we can essentially reverse the inequalities (30)-(34) and obtain the same results. In fact we have

Theorem 10. Equation (35) has at least one nontrivial solution if we assume $\ell>0$ and

$$
\begin{gathered}
\lambda_{\ell} t^{2} \leq 2 F(x, t)+W_{1}(x), \quad x \in \Omega, t \in \mathbb{R}, \text { for some } W_{1}(x) \in i L^{1}(\mathbb{R}), \\
2 F(x, t) \leq \lambda_{\ell} t^{2}, \quad|t| \leq \delta \text { for some } \delta>0 \\
2 F(x, t) \leq \nu t^{2}, \quad x \in \Omega, t \in \mathbb{R}, \text { for some } \nu<\lambda_{\ell+1} \\
H(x, t) \geq-C(|t|+1), \quad x \in \Omega, t \in \mathbb{R}
\end{gathered}
$$


and

$$
\liminf _{|t| \rightarrow \infty} H(x, t) /|t|>0 \quad \text { a.e. }
$$

Proof. In this case we take $G$ to be the functional (29). We take $A=N, N^{\prime}=$ $N \ominus\left\{v_{0}\right\}$, and consider the mapping

$$
F\left(v+w+s v_{0}\right)=v+\left[s+\delta-\delta \varphi\left(\|w\|^{2} / \delta^{2}\right)\right] v_{0}, \quad v \in N^{\prime}, w \in M, s \in \mathbb{R},
$$

where $\varphi$ satisfies the hypotheses of Proposition 5. By (43) we have

$$
G(v) \leq B_{1}, \quad v \in N .
$$

For $w \in M_{1}$ we write $w=w^{\prime}+y$, where $w^{\prime} \in M$ and $y \in E\left(\lambda_{\ell}\right)$. Then (44) implies

$$
G(w) \geq \epsilon_{1}, \quad\|w\|_{D}=\rho, w \in M_{1},
$$

unless (35) has a nontrivial solution. Hence by the argument given in the proof of Theorem 7 we have a sequence satisfying (39). If $\tilde{u}_{k}$ and $\tilde{u}$ are as in the proof of Theorem 7, then (46), (47) imply that $\tilde{u} \equiv 0$ as in that proof. However, (40) implies

$$
2 \int_{\Omega} F\left(x, u_{k}\right) d x / \rho_{k}^{2} \rightarrow 1
$$

while (45) implies

$$
\limsup 2 \int_{\Omega} F\left(x, u_{k}\right) d x / \rho_{k}^{2} \leq \nu \int_{\Omega} \tilde{u}^{2} d x,
$$

showing that $\tilde{u} \not \equiv 0$. This contradiction proves the theorem as in the case of Theorem 7 .

The proof of Theorem 10 implies

Corollary 11. If $\lambda_{\ell}$ is a simple eigenvalue, then hypothesis (43) can be weakened to

$$
\lambda_{\ell-1} t^{2} \leq 2 F(x, t)+W_{1}(x), \quad x \in \Omega, t \in \mathbb{R}, \text { for some } W_{1}(x) \in L^{1}(\mathbb{R}),
$$

in Theorem 10.

\section{Some Important QUANTITIES}

We now show that we can improve the results of the last section. For each fixed $k$, let $N_{k}$ denote the subspace of $D:=D\left(A^{1 / 2}\right)$ spanned by the eigenfunctions corresponding to $\lambda_{0}, \ldots, \lambda_{k}$, and let $M_{k}=N_{k}^{\perp} \cap D$. Then $D=M_{k} \oplus N_{k}$. We define

$$
\alpha_{k}:=\max \left\{(A v, v): v \in N_{k}, v \geq 0,\|v\|=1\right\},
$$

where $\|v\|$ denotes the $L^{2}(\Omega)$ norm of $v$. We assume that $A$ has an eigenfunction $\varphi_{0}$ of constant sign a.e. on $\Omega$ corresponding to the eigenvalue $\lambda_{0}$.

Next we define for $a \in \mathbb{R}$,

$$
\gamma_{k}(a):=\max \left\{(A v, v)-a\left\|v^{-}\right\|^{2}: v \in N_{k},\left\|v^{+}\right\|=1\right\}
$$

and

$$
\Gamma_{k}(a):=\inf \left\{(A w, w)-a\left\|w^{-}\right\|^{2}: w \in M_{k},\left\|w^{+}\right\|=1\right\},
$$

where $u^{ \pm}=\max \{ \pm u, 0\}$. 
We take any integer $\ell \geq 0$ and let $N$ denote the subspace of $L^{2}(\Omega)$ spanned by the eigenspaces of $A$ corresponding to the eigenvalues $\lambda_{0}, \lambda_{1}, \ldots, \lambda_{\ell}$. We take $M=N^{\perp} \cap D$, where $D=D\left(A^{1 / 2}\right)$. We assume that $F(x, t)$ satisfies

$$
\begin{gathered}
a_{1}\left(t^{-}\right)^{2}+\gamma_{\ell}\left(a_{1}\right)\left(t^{+}\right)^{2}-W_{1}(x) \leq 2 F(x, t) \\
\leq a_{2}\left(t^{-}\right)^{2}+\nu\left(t^{+}\right)^{2}, \quad x \in \Omega, t \in \mathbb{R},
\end{gathered}
$$

for numbers $a_{1}, a_{2}$ satisfying $\alpha_{\ell}<a_{1} \leq a_{2}$, where $W_{1}$ is a function in $L^{1}(\Omega)$ and $\nu<\Gamma_{\ell}\left(a_{2}\right)$. We also assume that

$$
\begin{gathered}
2 F(x, t) \leq \lambda_{\ell+1} t^{2}, \quad|t| \leq \delta \text { for some } \delta>0, \\
|f(x, t)| \leq C|t|+W(x), W \in L^{2}(\Omega), \\
f(x, t) / t \rightarrow \alpha_{ \pm}(x) \text { a.e. as } t \rightarrow \pm \infty
\end{gathered}
$$

and the only solution of

$$
A u=\alpha_{+}(x) u^{+}-\alpha_{-}(x) u^{-}
$$

is $u \equiv 0$. We have

Theorem 12. Under the above hypotheses, (35) has a nontrivial solution.

Proof. By (50),

$$
\|v\|_{D}^{2} \leq a_{1}\left\|v^{-}\right\|^{2}+\gamma_{\ell}\left(a_{1}\right)\left\|v^{+}\right\|^{2}, \quad v \in N
$$

and by (51) we have

$$
a_{2}\left\|w^{-}\right\|^{2}+\Gamma_{\ell}\left(a_{2}\right)\left\|w^{+}\right\|^{2} \leq\|w\|_{D}^{2}, \quad w \in M .
$$

Hence

$$
G(v) \leq B_{1}, \quad v \in N .
$$

Since $\nu<\Gamma_{\ell}\left(a_{2}\right)$, we see by continuity that there is an $\varepsilon>0$ such that

$$
\nu<(1-\varepsilon) \Gamma_{\ell}\left(\frac{a_{2}}{1-\varepsilon}\right) .
$$

Hence,

$$
\begin{aligned}
G(w) & \geq \varepsilon\|w\|_{D}^{2}+(1-\varepsilon)\left[\Gamma_{\ell}\left(\frac{a_{2}}{1-\varepsilon}\right)-\frac{\nu}{1-\varepsilon}\right]\left\|w^{+}\right\|^{2} \\
& \geq \varepsilon\|w\|_{D}^{2}, \quad w \in M
\end{aligned}
$$

by $(52)$.

As in the proof of Theorem 7 , we note that the following alternative holds:

Either

(a) there is an infinite number of eigenfunctions $y \in E\left(\lambda_{\ell}\right) \backslash\{0\}$ such that

$$
A y=f(x, y)=\lambda_{0} y
$$

or

(b) for each $\rho>0$ sufficiently small, there is an $\epsilon>0$ such that

$$
G(w) \geq \epsilon, \quad\|w\|_{D}=\rho, w \in M_{\ell} .
$$


Since option (a) solves our problem, we may assume that option (b) holds. Let $v_{0} \in E\left(\lambda_{\ell}\right)$, and let $F$ be the mapping (17). Take $A=N, B=F^{-1}\left(\delta v_{0}\right)$. By (59), (60), (62) we see that (7) holds with $b_{0}>0$ and $a_{0}=B_{1}$. By Proposition 5 we can conclude that there is a sequence $\left\{u_{k}\right\} \subset D$ such that

$$
G\left(u_{k}\right) \rightarrow c, b_{0} \leq c \leq B_{1}, G^{\prime}\left(u_{k}\right) \rightarrow 0 .
$$

Thus

$$
G\left(u_{k}\right)=\left\|u_{k}\right\|_{D}^{2}-2 \int_{\Omega} F\left(x, u_{k}\right) d x \rightarrow c
$$

and

$$
\left(G^{\prime}\left(u_{k}\right), v\right)=2\left(u_{k}, v\right)_{D}-2\left(f\left(u_{k}\right), v\right) \rightarrow 0, \quad v \in D .
$$

If $\rho_{k}=\left\|u_{k}\right\|_{D} \rightarrow \infty$, let $\tilde{u}_{k}=u_{k} / \rho_{k}$. Then $\left\|\tilde{u}_{k}\right\|_{D}=1$. Thus there is a renamed subsequence such that $\tilde{u}_{k} \rightarrow \tilde{u}$ weakly in $D$, strongly in $L^{2}(\Omega)$ and a.e. in $\Omega$. Hence

$$
G\left(u_{k}\right) / \rho_{k}^{2}=\left\|\tilde{u}_{k}\right\|_{D}^{2}-2 \int_{\Omega} F\left(x, u_{k}\right) d x / \rho_{k}^{2} \rightarrow 0
$$

Since

$$
\left|F\left(x, u_{k}\right)\right| / \rho_{k}^{2} \leq C\left(\left|\tilde{u}_{k}(x)\right|^{2}+W_{3}(x) / \rho_{k}^{2}\right), \quad W_{3} \in L^{1}(\Omega),
$$

by (52), and the right hand side of (66) converges to $C|\tilde{u}(x)|^{2}$ in $L^{1}(\Omega)$ and

$$
2 F\left(x, u_{k}(x)\right) / \rho_{k}^{2} \rightarrow \alpha_{+}(x)\left(\tilde{u}^{+}\right)^{2}+\alpha_{-}(x)\left(\tilde{u}^{-}\right)^{2} \quad \text { a.e. },
$$

we see that the convergence in $(67)$ is not only pointwise a.e. but also in $L^{1}(\Omega)$. Since $\left\|\tilde{u}_{k}\right\|_{D}=1,(66)$ implies

$$
\int_{\Omega}\left\{\alpha_{+}\left(\tilde{u}^{+}\right)^{2}+\alpha_{-}\left(\tilde{u}^{-}\right)^{2}\right\} d x=1 .
$$

Also

$$
\left(G^{\prime}\left(u_{k}\right), v\right) / \rho_{k}=2\left(\tilde{u}_{k}, v\right)_{D}-2\left(f\left(u_{k}\right), v\right) / \rho_{k} \rightarrow 0
$$

for each $v \in D$. This implies

$$
(\tilde{u}, \tilde{v})_{D}=\left(\alpha_{+} \tilde{u}^{+}-\alpha_{-} \tilde{u}^{-}, v\right), \quad v \in D .
$$

Consequently, $\tilde{u}$ is a solution of (56). By hypothesis $\tilde{u} \equiv 0$. But this contradicts (68). Hence $\rho_{k} \leq C$. The theorem now follows from Theorem 3.4.1 of [Sc4].

\section{REFERENCES}

[Ad] R. A. Adams, Sobolev Spaces, Academic Press, 1975. MR0450957 (56:9247)

[BL] J. Bergh and J. Löfström, Interpolation Spaces, Springer, 1976. MR0482275 (58:2349)

[FMS] M.F. Furtado, L.A. Maia, and E.A.B. Silva, On a double resonant problem in $\mathbb{R}^{N}$. Differential Integral Equations 15 (2002), no. 11, 1335-1344. MR1920690 (2003g:35064)

[FS] M.F. Furtado and E.A.B. Silva, Double resonant problems which are locally non-quadratic at infinity. Proceedings of the USA-Chile Workshop on Nonlinear Analysis (Viña del MarValparaiso, 2000), 155-171 (electronic), Electron. J. Differ. Equ. Conf., 6, Southwest Texas State Univ., San Marcos, TX, 2001. MR1804772 (2002g:35079)

[Sc1] M. Schechter, New saddle point theorems. Generalized functions and their applications (Varanasi, 1991), 213-219, Plenum, New York, 1993. MR1240078 (94i:58034)

[Sc2] M. Schechter, A generalization of the saddle point method with applications, Ann. Polon. Math. 57 (1992), no. 3, 269-281. MR1201854 (94c:58028)

[Sc3] M. Schechter, New linking theorems, Rend. Sem. Mat. Univ. Padova, 99(1998) 255-269. MR1636619 (99h:58035) 
[Sc4] M. Schechter, Linking Methods in Critical Point Theory, Birkhäuser Boston, 1999. MR1729208 (2001f:58032)

[Si1] E. A. de B e Silva, Linking theorems and applications to semilinear elliptic problems at resonance, Nonlinear Analysis TMA 16(1991), 455-477. MR1093380 (92d:35108)

Department of Mathematics, University of California, Irvine, California 92697-3875

E-mail address: mschecht@math.uci.edu 